\title{
CLASSICAL CONTROL OF QUANTIZED FIELDS: CAVITY QED AND THE PHOTON PISTOL
}

\author{
J.H. EBerLy AND C.K. LAW \\ Department of Physics and Astronomy \\ and \\ Rochester Theory Center for Optical Science and Engineering \\ University of Rochester \\ Rochester, NY 14627, USA
}

\begin{abstract}
By using the double-lambda configuration of energy levels and three classical fields, an atom or ion in a high- $Q$ resonant cavity can be manipulated to create a single-mode quantum state in the cavity that is an arbitrary combination of Fock states for that mode. The same principles of operation can be applied to a cavity with much lower $Q$ in order to produce a "photon pistol", a device that "shoots" one and only one photon when a trigger is pulled, and emits no photons at other times.
\end{abstract}

PACS numbers: $42.50 . \mathrm{Dv}, 42.50 . \mathrm{Ct}, 32.80 . \mathrm{Qk}$

Cavity QED now provides a realistic framework for experimental study of strongly interacting quantum systems, and for the production of interesting varieties of non-classical states of atoms and photons. It is almost too easy in the context of cavity QED to produce hyper-quantum states, states that are extremely tangled combinations of mixed numbers of atoms and photons. It is not usually realized that in 1958 Jaynes already predicted [1] that cavity QED would provide an attractive way to study entanglement.

Here we address a related question that has captured some attention in the past few years but has proved difficult to answer with complete satisfaction. This is the question how best to produce arbitrary, pre-specified, quantized cavity-mode states without entanglement with atomic states, even without entanglement of the atom used to generate the photons, and to do so using classically controllable inputs.

What classical device or collection of classical devices would be suitable to generate an arbitrary non-entangled quantum state with sufficient reliability that it could serve as the heart of a photon factory? Such a factory could, for example, act as supplier for a shop such as the one shown in Fig. 1. Or, if the engine were compact and reliable enough, perhaps Fock \& Associates could install one in the cellar of their shop and run their own factory. At the present time there are three 


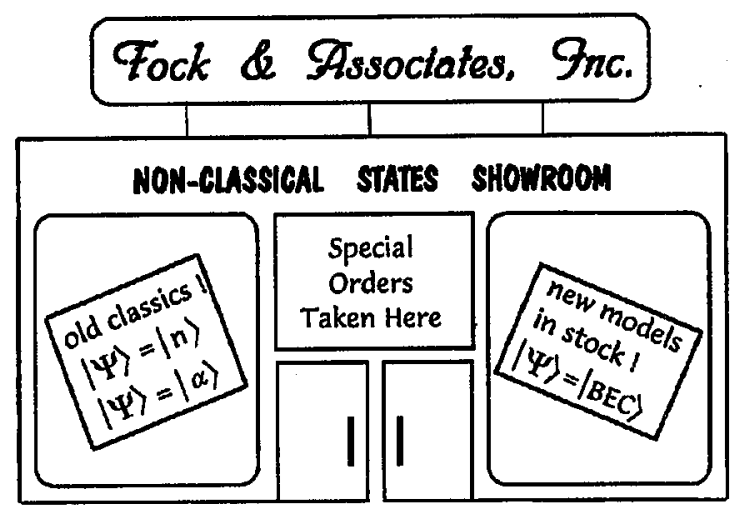

Fig. 1. A retail showroom where quantum states are available.

models for a photon engine capable, in principle, of generating an arbitrary state of the cavity mode, say

$$
|\Psi\rangle=\sum_{n=0}^{N} c_{n}|n\rangle
$$

where the coefficients $c_{n}$ are to be specified by the customer and must satisfy probability conservation, $\sum\left|c_{n}\right|^{2}=1$, but are otherwise arbitrary, and where $N$ is the highest occupation number desired.

These model engines are sketched in Fig. 2. Model 1 is an "exit control" model [2]. It uses extremely low-cost raw materials, just a stream of identically prepared two-level atoms, but it requires a rigorous quality control stage, because the action of the atoms inside the cavity is fully quantum mechanical and the cavity builds up to its desired state only after the passage of at least $N$ atoms, and typically many more than that. The state of each atom must be monitored when it leaves the cavity to determine if the cavity state is on the right path to the desired result. Because of the statistical rarity of achieving the desired output and the overtime wages of the quality control inspector, this is a low-profit model.

Model 2 is an "input control" model [3]. It can produce a desired output with commendable reliability, but it consumes very expensive raw materials. The raw materials required are carefully tailored quantum systems with imprinted coherence properties, for example a distribution of properly sequenced probability amplitudes in a Zeeman multiplet. Under the right conditions in the cavity, this coherence can be transformed into a coherence property of the cavity mode, yielding the desired cavity state directly, with only a cursory inspection of the output to make sure the transformation has occurred. Because of the expense of producing the carefully tailored raw materials (and drastically different tailoring for every customer must be anticipated), this is also a low-profit model.

Model 3 is a "process control" model [4]. It uses even less expensive raw materials than model 1 because it needs only a single atom, and it requires minimal quality control. However, it uses a carefully programmed interaction sequence during the time the atom is in the cavity. This means that external classical fields 


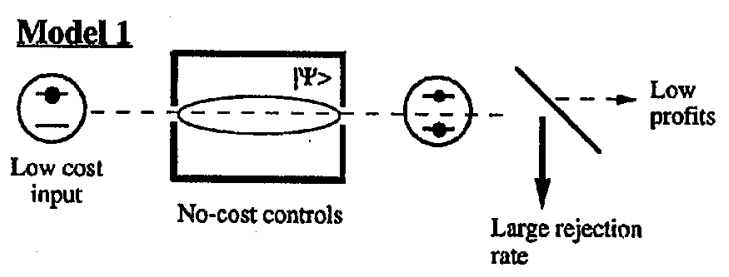

Model 2
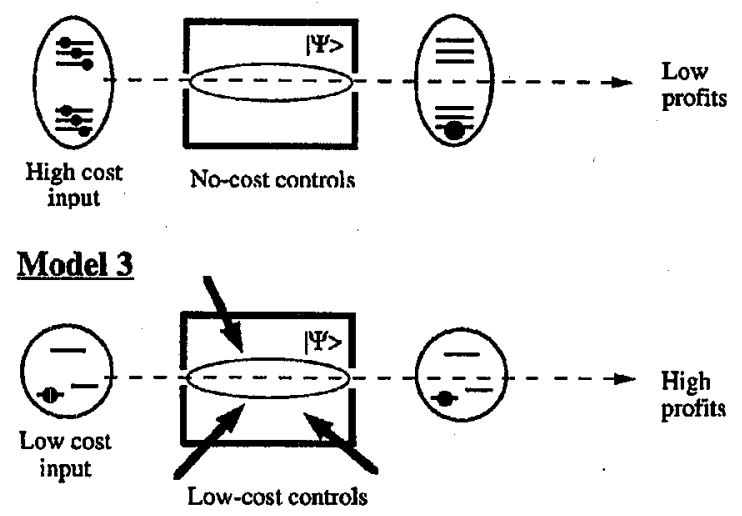

Fig. 2. Sketches of three models that have been proposed for a photon engine that will generate photon states according to a customer's prescription.

are used to excite and de-excite the atom in a predetermined sequence of steps. The major cost is not in supplying such external fields but in building a cavity that permits these fields to enter and leave without seriously affecting the $Q$ value of the quantized cavity mode of interest: This is a capital cost that needs to be undertaken only once, independent of the state that is to be produced. Thus a factory using model 3 needs sufficient business volume to recover the capital cost of the unusual cavity, but is otherwise a low-cost and high-profit model.

The odds appear to be in favor of model 3 , so we will now explain its advantages more carefully. One important difference that was not mentioned explicitly, although it is implied by the sketch in Fig. 2, is that the atom is used in a stimulated Raman mode, which means that the two-level atom is really a three-level atom being excited and de-excited very far off resonance in a lambda configuration. Moreover, the lambda interaction is employed twice, so the atom is actually a "double-lambda" atom, as shown in Fig. 3.

The absorption-emission events are those of two Raman-type processes acting between the same two levels of an atom at the same time: absorption of a pump photon and the accompanying emission of a Stokes photon, followed by the absorption of another pump photon and then the emission of an anti-Stokes photon, and in the figure $R$ and $L$ denote the "right" and "left" pump modes, while $S$ and $A$ label the usual Stokes and anti-Stokes modes. In an alternating-pair sequence these fields can take the atom from state $|-\rangle$ to state $|+\rangle$ and around to $|-\rangle$ again. 


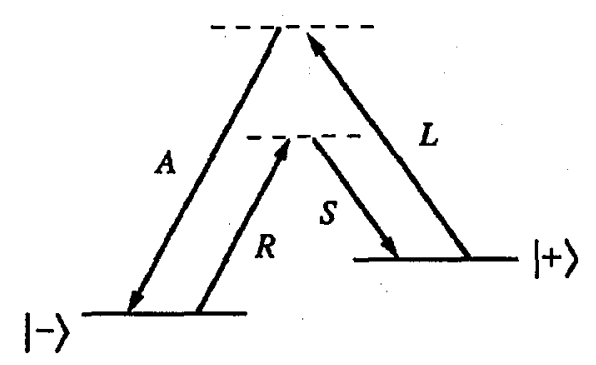

Fig. 3. Sketch of double-lambda atom. Dipole transition matrix elements connect the two "ground" states with an upper opposite-parity state that is not shown.

In this way, the atom ends where it began, but the field inventory of photons is different. In the end there are two fewer pump photons and one additional photon in each of the Stokes and anti-Stokes modes. The atom has acted as a wave-mixing catalyst, and is ready to be used again. The Jaynes-Cummings system has only a single channel connecting its two states, so cyclic operation of this kind is not possible.

The double-lambda atom has not received a lot of attention in the literature, but there are applications for which its advantages have already been recognized. Some of the previous works can be identified with the following themes and names:

(i) amplification without inversion, Kocharovskaya and Mandel [5];

(ii) excitation via a classical and a quantum channel simultaneously, Law and Eberly [6];

(iii) exact quantum solutions for 3 modes, Wang et al. [7];

(iv) boson spin algebra for 4 modes, Wang and Eberly [8];

(v) two mechanisms for inversionless amplification, Keitel et al. [9];

(vi) perfect Greenberger-Horne-Zeilinger (GHZ) correlator, Wodkiewicz et al. [10];

(vii) two-mode squeezing and phase correlation, Law and Eberly [11];

(viii) lasing without inversion and phaseonium, Scully et al. [12];

(ix) transparency and dressing in a double-lambda medium, Cerboneschi and Arimondo [13];

(x) photon engine, Law and Eberly [4];

(xi) quantum image storage, Kneer and Law [14];

(xii) photon pistol, Law and Kimble [15].

The double-lambda is obviously a two-channel extension of the well-known Jaynes-Cummings (JC) model [1]. One knows that the JC model is essentially a handy toy in which to examine fundamental aspects of both optical spectroscopy and laser action, with the major advantage that strong-field cavity interactions of current experimental interest can be treated in detail. However, many effects and processes familiar in optical physics do not fit into the Jaynes-Cummings framework at all. These include: 
(a) pump-probe effects,

(b) nonlinear wave mixing, including down conversion,

(c) three-mode correlations of the GHZ type,

(d) two-photon lasing, and

(e) optical pumping of laser action.

Because of its two channels, the double-lambda model is able to treat some or all of these effects. The main important difference to the JC model is the ability to manipulate the atom by connecting the states $|+\rangle$ and $|-\rangle$ in two independent ways. This has been shown [4] to be the key to avoid entanglement. Although a 3-mode quantum model of the two-channel process also exists [7], we are concerned here only with a 4-mode model [8]. In any case a single atom (or ion) is either laser-cooled and allowed to drift very slowly through the cavity or it is permanently trapped in the cavity. Figure 4 sketches the three (classical) light bearns incident from outside the cavity that are imposed to control atomic excitation in either case.
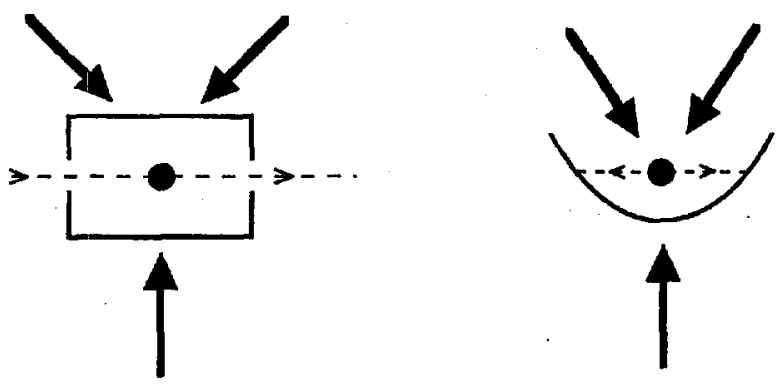

Fig. 4. Arrows designate three classical external radiation fields (laser beams) that are directed at a double-lambda atom that drifts through a cavity or is held in a trap, which is located in a cavity too. The cavity supports excitation of a fourth mode, which is excited one photon at a time and is treated as quantized.

It is remarkable that the much more complex quantum double-lambda is "solvable" in the same sense as the JC model - the eigenvalues and eigenfunctions of its Hamiltonian can be found exactly. For comparison, the two models' interaction Hamiltonians are

$$
\begin{aligned}
& V_{\mathrm{JC}}=g \hat{a} \hat{\sigma}_{+-} \text {h.c., } \\
& V_{\Lambda-\Lambda}=g\left(\hat{a}_{R} \hat{a}_{S}^{+}+\hat{a}_{L}^{+} \hat{a}_{A}\right) \hat{\sigma}_{+-}+\text {h.c. }
\end{aligned}
$$

It is well known that the raising and lowering operators for the JC model atom have an angular momentum interpretation. In the 3-mode case Schwinger's procedure extends an angular momentum property to the radiation mode operators. For example, we define $L_{1}$ operators using bilinear combinations of the $R$ and $S$ modes: $L_{1+} \equiv a_{R}^{+} a_{S}$ and $L_{1-} \equiv a_{R} a_{S}^{+}$, and $L_{1 z} \equiv \frac{1}{2}\left[L_{1+}, L_{1-}\right]$. Then $L_{2}$ operators are formed from the $A$ and $L$ mode operators in the same way. The 
connections between angular momentum quantum numbers and photon numbers are given by

$$
l_{1}=\frac{1}{2}\left(n_{R}+n_{S}\right), \quad m_{1}=\frac{1}{2}\left(n_{R}-n_{S}\right),
$$

and

$$
l_{2}=\frac{1}{2}\left(n_{A}+n_{L}\right), \quad m_{2}=\frac{1}{2}\left(n_{A}-n_{L}\right) .
$$

These angular momenta, plus the atom's two-level pseudo-spin $S$, are used to define a new "total" angular momentum $\boldsymbol{J}=\boldsymbol{L}_{1}+\boldsymbol{L}_{2}+\boldsymbol{S}$. It has been shown [8] that the eigenvalues and eigenfunctions can be found for this Hamiltonian exactly and analytically in closed form. (The same is true for the 3-mode double-lambda systems [7], but angular momentum algebra is not helpful there and the results are strikingly different.) In the 4-mode case of interest here we only quote the eigenvalue formula, which is given by a Clebsch-Gordan coefficient

$$
\lambda=\sqrt{(l-m)(l+m+1)},
$$

where $l$ and $m$ belong to the "mode angular momentum" $L=L_{1}+L_{2}$.

Surprisingly, the most interesting applications of the double-lambda model are not even on our list of effects lying outside the reach of the JC model. These are the consequences of understanding the working of a photon "engine", and one of these is the invention of a "photon pistol."

Only the design for the photon engine [4] has been published so far. In the case of the photon pistol, the same combination of external and cavity modes is used as for the engine, but the cavity $Q$ is drastically lowered so that as soon as a cavity photon is generated in the cycling of the "engine", it is transmitted through one of the cavity mirrors ("shot") out of the cavity. A second photon will not be emitted until the engine is run through another cycle. Since the principle of the pistol is clear, the remaining issue is only one of practicality. Surprisingly, the device is not impractical and a photon pistol can probably be made in the laboratory in the near future.

Here we list the results of a study of a photon pistol in the context of the $D 1$ line of Cs. This turns out to provide a particularly attractive context for pistol operation. The relevant $\mathrm{Cs}$ transitions for this are shown in Fig. 5. The $F=4 \rightarrow F=4$ transition in Fig. 5 is the one we have labelled $R$ above, and $r_{0}$ is its peak Rabi frequency. The $F=4 \rightarrow F=3$ transition is the one labeled $S$ above, and $g$ is its one-photon Rabi frequency. The "recycling" transitions involving the external fields labeled $L$ and $A$ above, that make up the other half of the double lambda are omitted from the figure for simplicity.

Some of the key operating points are these. Once an atom is chosen, the value of $g$ is fixed according to the relevant dipole moment, the size of the cavity, and the position of the atom in it. The other transitions in the atom determine how strong $r_{0}$ can be made before higher excitations need to be considered. In the Cs case this means the critical parameter, the effective two-photon Rabi frequency $\Omega_{R S}$ given by

$$
\Omega_{R S}=\frac{1}{2} \frac{g r_{0}}{\Delta}
$$




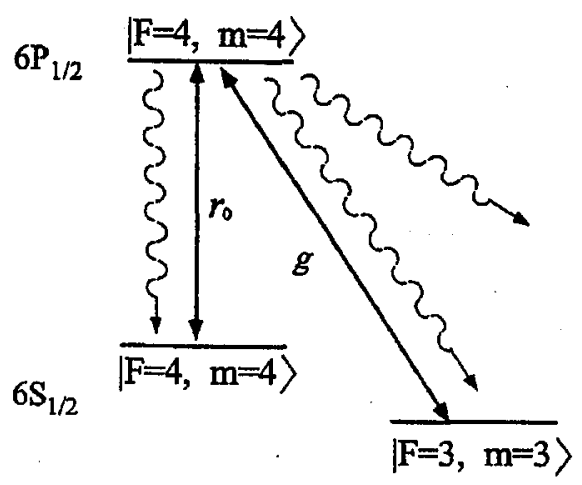

Fig. 5. The basis for operation of the photon pistol is the induced appearance of a photon in the cavity (channel " $g$ " here). The relevant energy levels of the experimental atom (Cs operating on the $D 1$ line) are shown, along with the principle atomic relaxation channels. The cavity relaxation is much more rapid, allowing the photon "bullet" to emerge from the pistol with less than $0.1 \mu$ s delay.

is too weak to be effective unless $\Delta$, the one-photon detuning, is nearly resonant. This has no effect on the pistol concept, but does allow the upper state $(m=4$ in the figure) to become populated, thus opening an extra relaxation channel (spontaneous emission from $m=4$ ) disregarded up to now. Both quantum trajectory calculations and also more elaborate master equation calculations [15] show that this effect can be tolerated.

At resonance, the following parameter values are currently realizable in the laboratory for the Cs transitions shown: ( $r_{0}, g$, the cavity loss rate $\kappa$ and the atomic decay rate $\gamma)=(22.5,45,45,4.5)$, where all values are in $\mathrm{MHz}$. To be specific, $\kappa=$ $2 \pi \cdot 45 \mathrm{MHz}$, etc. Given a temporal halfwidth $T_{0}=3 \gamma^{-1}=105 \mathrm{~ns}$ for the external field $R$, calculations that take full account of hyperfine structure, atomic decay channels, and operating cavity conditions lead to the following conclusions [15]. The one-photon emission probability rises more than $95 \%$ within less than $0.1 \mu \mathrm{s}$, just two atomic lifetimes $(\gamma t=2)$. An even higher emission probability $(99.5 \%)$ can be reached when $g=2 \pi \cdot 120 \mathrm{MHz}$. In the absence of another $R$ pulse there will be no second photon. This is an operational reliability that is probably acceptable to most customers (quantum optics Mafia, etc.). An important selling point is that the fall-off in performance is not great even for a significant decrease in reliability of input. For example, if $g$ fluctuates by a factor of 2 it is predicted that the firing probability still remains near to, or well above, $90 \%$.

In summary, we have discussed progress toward classical control of quantum processes. The two-channel double-lambda system has been exploited here to provide two examples of classical control, the photon state engine and the photon pistol. Both rely on very highly lossless operation of an optical cavity of very small dimension. Until recently these requirements would have been unrealistic, and at this time they remain just beyond realization for the photon engine. However, the photon pistol requires a much lower storage time for the cavity mode, and we have reported the results of a recent calculation [15] to show that a pistol is prob- 
ably feasible at the present time. Just as interesting are the items in the second listing above. They represent targets for future application of the double-lambda principle.

\section{Acknowledgment}

The research reported here was supported by the US National Science Foundation through grants PHY94-08733 and PHY94-15583.

\section{References}

[1] E.T. Jaynes, Microwave Lab Report 502, Stanford Univ., 1958; see also E.T. Jaynes, F.W. Cummings, Proc. IEEE 51, 89 (1963).

[2] K. Vogel, V.M. Akulin, W.P. Schleich, Phys. Rev. Lett. 71, 1816 (1993); B.M. Garraway, B. Sherman, H. Moya-Cessa, P.L. Knight, G. Kurizki, Phys. Rev. A 49, 535 (1994); for a model using a single conditional measurement, see A. Kozhekin, G. Kurizki, B. Sherman, Phys. Rev. A 54, 3535 (1996).

[3] A.S. Parkins, P. Marte, P. Zoller, H.J. Kimble, Phys. Rev. Lett. 71, 3095 (1993); A.S. Parkins, P. Marte, P. Zoller, O. Carnal, H.J. Kimble, Phys. Rev. A 51, 1578 (1995).

[4] C.K. Law, J.H. Eberly, Phys. Rev. Lett. 76, 1055 (1996).

[5] O. Kocharovskaya, P. Mandel, Phys. Rev. A 42, 523 (1990).

[6] C.K. Law, J.H. Eberly, Phys. Rev. A 43, 6337 (1991).

[7] L. Wang, R.R. Puri, J.H. Eberly, Phys. Rev. A 46, 7192 (1992).

[8] L. Wang, J.H. Eberly, Phys. Rev. A 47, 4248 (1993).

[9] C.H. Keitel, O.A. Kocharovskaya, L.M. Narducci, M.O. Scully, S.-Y. Zhu, H.M. Doss, Phys. Rev. A 48, 3196 (1993).

[10] K. Wodkiewicz, L. Wang, J.H. Eberly, Phys. Rev. A 47, 3280 (1993).

[11] C.K. Law, J.H. Eberly, Phys. Rev. A 47, 3195 (1993).

[12] M. Fleischhauer, M.D. Lukin, D.E. Nikonov, M.O. Scully, Opt. Commun. 110, 351 (1994).

[13] E. Cerboneschi, E. Arimondo, Phys. Rev. A 52, R1823 (1995).

[14] B. Kneer, C.K. Law, Paper MEE7, Annual Meeting, Optical Society of America, Rochester, NY, October 20-24, 1996.

[15] C.K. Law, H.J. Kimble, J. Mod. Opt., special issue on Quantum States Preparation and Measurement, Eds. W.P. Schleich, M.G. Raymer, in press. 